\title{
A case of subtrochanteric femur fracture nonunion with failed implant in situ treated with exchange nailing using interlock nail and autologous bone grafting: a case report
}

\author{
Neetin P. Mahajan*, Pranay Kondewar, Lalkar Gadod, Amey Sadar, Shubham Atal
}

Department of Orthopedics, JJ Hospital, Mumbai, Maharashtra, India

Received: 27 May 2021

Accepted: 15 June 2021

\section{*Correspondence:}

Dr. Pranay Kondewar,

E-mail: pranaypk1@gmail.com

Copyright: ( $)$ the author(s), publisher and licensee Medip Academy. This is an open-access article distributed under the terms of the Creative Commons Attribution Non-Commercial License, which permits unrestricted non-commercial use, distribution, and reproduction in any medium, provided the original work is properly cited.

\begin{abstract}
Subtrochanteric femur fracture accounts for $25 \%$ of all hip fracture and may land up in non-union due to the inadequate reduction and fixation tech, local muscle pull over fragments, biomechanical stress in subtrochanteric region and soft tissue interposition etc., non-union are managed with various choices of implants like exchange nailing, angle blade plate, dynamic condylar screw, augmentation of previous hardware with plate and by providing biological environments at fracture site using bone graft. Strict adherence to principles of providing stability to fracture and providing environment for bony growth gives good clinical outcome. A 52 years old male with subtrochanteric femur fracture was operated with long PFN, later presented to us after 18 months with failure of the hardware and atrophic non-union manifesting as pain during walking and limping. Patient was operated with removal of implant and exchange nailing using femur interlock nail and autologous bone grafting from iliac crest graft. 1 year follow up showed complete bony union and abundant of callus formation. Patient is currently doing all the daily activities and have no complaints at present. At 1 year follow up there is complete union at non-union site and good clinical outcome is achieved. Exchange nailing with interlock nail and autologous bone grafting for treatment of atrophic non-union of subtrochanteric femur fractures gives good clinical outcome.
\end{abstract}

Keywords: Subtrochanteric femur fracture, Non-union, Exchange nailing, Mis a nail technique, Bone grafting, Femur interlock nail

\section{INTRODUCTION}

Subtrochanteric femoral fractures account for approximately $25 \%$ of all hip fractures and have a bimodal age and sex distribution. ${ }^{1}$ The subtrochanteric region is defined as the area between the lesser trochanter and the femoral isthmus, which is five centimetres below the trochanter. ${ }^{2}$ The femur is exposed to biomechanical forces due to osseous anatomic conditions in the region and the muscles surrounding the hip joint. Shear forces affect the cancellous bone of the proximal femur, whereas bending forces especially affect the cortical bones of the subtrochanteric area and the shaft. $^{3}$ Seinsheimer classification and Russel Taylor classification system are used clinically. Non-union are because of improper reduction, soft tissue interposition and infection post op. ${ }^{4}$

Non-unions of the subtrochanteric region of the femur after previous intramedullary nailing can be difficult to address. Implant failure and bone defects around the implant significantly complicate the therapy, and complex surgical procedures with implant removal, extensive debridement of the non-union site, bone grafting and reosteosynthesis usually become necessary.

Revision surgery for non-union is mostly preferred with angle blade plates, PF-LCP (proximal femur locking plate), dynamic condylar screw or exchange nailing with 
proximal femur nail (PFN) also gives internal support and rigidity to construct. Bone grafting is invariably necessary in cases of non- union, allograft plus autograft is generally used.

\section{CASE REPORT}

A 52 years old male presented with complaints of pain in left hip after a fall from ladder since 2 days, patient was operated for left femur subtrochanteric fracture 18 months back and was walking with limp and pain since then. On local examination there was tenderness and swelling over hip, there was no scar tenderness or any discharge from previous surgery wound.

On X-ray of left hip non-united subtrochanteric femur fracture with broken long PFN and encirclage wire was noted. Blood investigation to rule out the infection was done and were normal. Patient was planned for exchange nailing surgery using interlock nail of bigger size and iliac bone grafting at the nonunion site. Patient was operated on traction table under spinal and epidural anaesthesia.

At first proximal 2 screws and one distal most screw along with encircled wire was removed. Then proximal part of the broken nail was taken out using implant removal set. To remove distal part of broken nail corticotomy window was created at a point below the fracture site, now distal broken part was held tightly, distal most screw removed and nail was removed from corticotomy site with backslap hammer after adducting the extremity.

Non-union site was cleared from fibrotic tissue, bony ends freshened and one dead bony fragment removed, now a larger size $11 \mathrm{~mm}$ femur interlock nail was inserted and fixed with 2 proximal and 2 distal screws, as proximal screws were near the fracture site additional 2 screws put by Miss a nail technique. One anterior and one posterior to nail to give additional stability to construct. Iliac crest bone grafting was done and void filled circumferentially, mainly on medial side of nonunion.

Patient was followed up monthly and was kept nil weight bearing for 10 weeks. After 10 weeks partial weight bearing started with toe touch walking. For next 2 months. 6 months X-ray showed tendency of bone towards union by callus formation around fracture site. Later full at bearing started and 1 year follow up showed abundance of callus and complete bony union. Patient is currently doing all the daily activities including running and jumping and has no complaints.

Exchange nailing with interlock nail and autologous bone grafting for treatment of atrophic non-union of subtrochanteric femur fractures is based on principles of non-union treatment, it gives stability to fractured bone and provide biological environment for bone union and gives good clinical outcome.

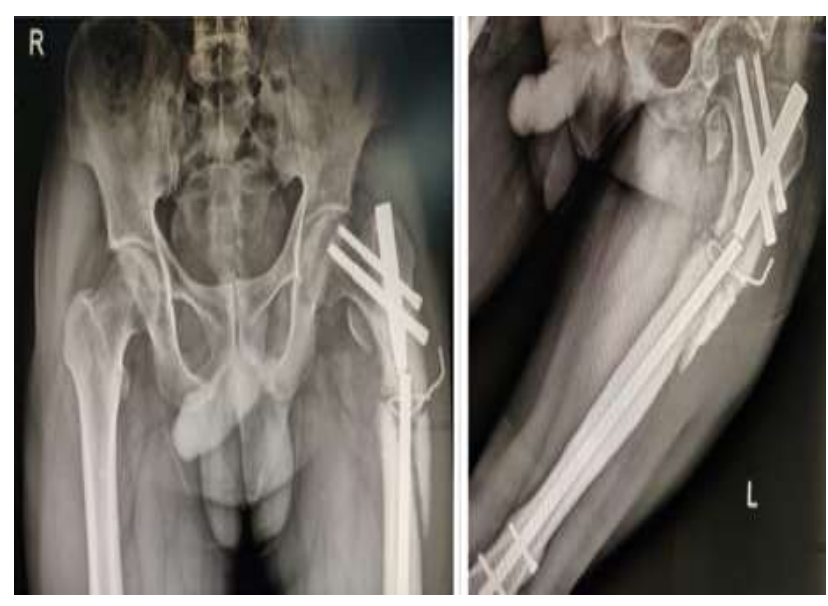

Figure 1: X-ray of pelvis and femur showing atrophic nonunion of subtrochanteric fracture with broken implant in situ, bony ends are sclerotic.

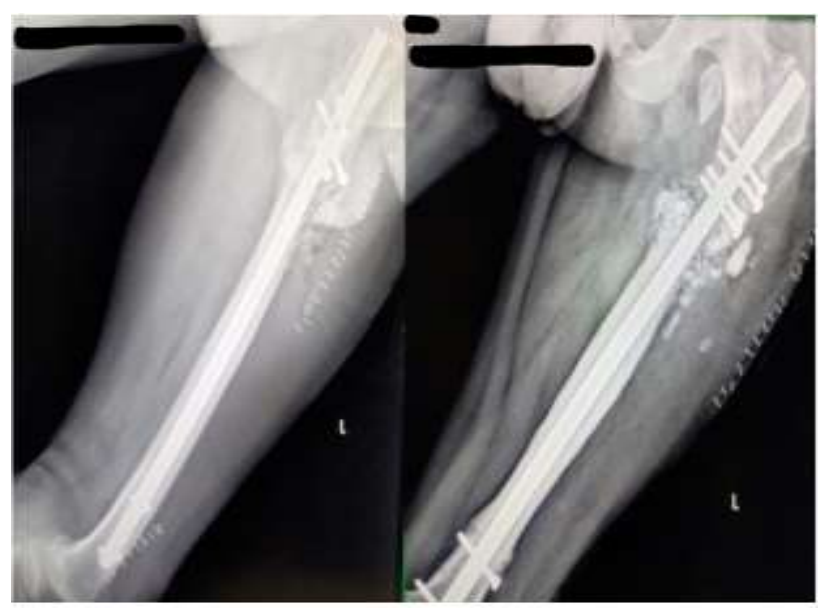

Figure 2: Immediate post op X-ray of femur, lateral and anteroposterior view interlock nail used to fix the fracture with miss a nail screws proximally and bone grafting done.
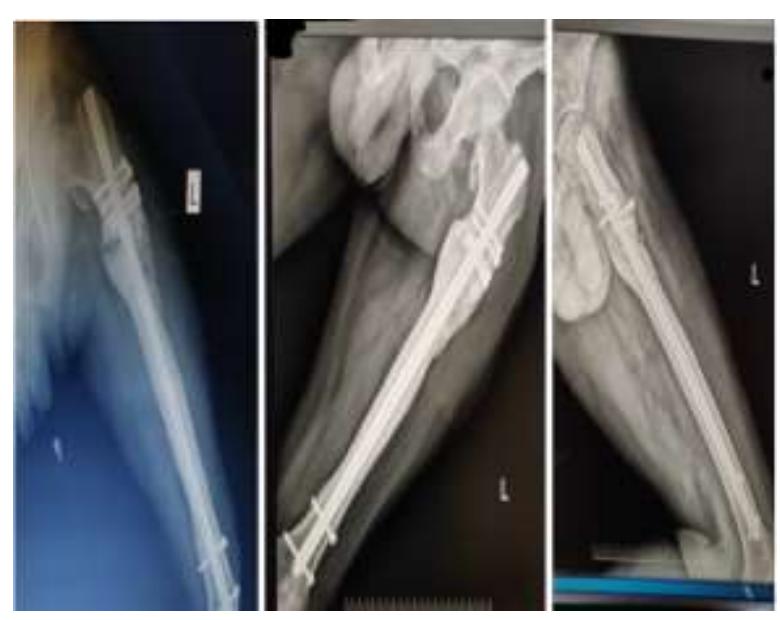

Figure 3: Follow up X-ray of femur showing bony union at 6 months and 12 months post op. 


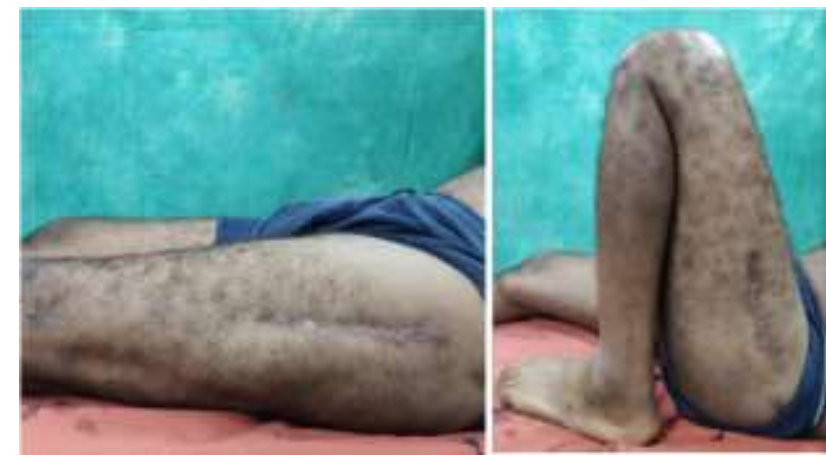

Figure 4: Clinical photographs of the patients left limb in full hip extension and flexion.

\section{DISCUSSION}

The management of subtrochanteric fractures is a challenging and technically demanding endeavour for surgeons. The fracture can be highly displaced and comminuted, because of the high concentration of stress in this area. The deforming forces for subtrochanteric fractures include flexion and external rotation from iliopsoas, abduction from the gluteal medius, adduction and shortening of the shaft from the hamstrings and the adductor muscle group.

The conventional and preferred treatment of subtrochanteric fractures has been intra medullary devices. They are load sharing devices which allow early weight bearing. several potential advantages, including a shorter lever arm (decreases tensile strain on implant) and efficient load transfer capacity. Biomechanically, considerable stress is applied to these fractures during body-weight loading, and because they also involve cortical bone, which has poor ability to achieve union, the time to union is relatively long, with a high risk of non-union or implant failure. ${ }^{5,6}$ Favourable mechanical characteristics of interlocking nails have eliminated the requirement of surgically reconstituting the medial femoral cortex, though extra medullary and intra medullary devices have been used to treat trochanteric fractures; each has its merits and demerits. $^{7,8}$

According to the current literature, there is no strong evidence to support the use of either IMN or extramedullary devices in the revision of subtrochanteric non-unions. Despite the development of new implants and increasing knowledge of non-union, the treatment of postoperative complications and persisting non-union still presents a challenge. To date, no prospective randomised study has been published; therefore, level IV studies are currently the best available evidence. ${ }^{9}$

There are previous reports in literature stating keeping the implant as it is when it's intact and additional plate support gives union but comparative studies of exchange nailing versus augmenting previous fixation with plate shows superiority of exchange nailing in terms of recovery time and healing achieved. ${ }^{10,11}$
Miss a nail technique is used in treatment of femoral neck fracture with additional shaft fractures, here we have modified it to use as an extra support to construct.

Simultaneous optimisation of the mechanical and biological environment is a successful method for managing complex subtrochanteric atrophic non-unions with failed implant. ${ }^{12}$ The diamond concept, include optimization of the mechanical environment along with the enhancement of the multidimensional biological pathways of bone healing, has been proposed as the framework of a single-stage surgical revision for the recalcitrant problem of atrophic non-unions with implant failure. $^{13}$

Bone grafting in form of combination of allograft and autograft alone or with additional growth factor like BMP7, hydroxyapatite and derivatives and RIA products have tried success. ${ }^{14}$

\section{CONCLUSION}

We have performed this surgery with consideration of providing adequate mechanical stability using larger sized interlock nail $(11 \mathrm{~mm})$ and additional proximal screw fixation using miss a nail technique and creating biological bed for bone growth that is cancellous allograft from iliac crest.

Funding: No funding sources

Conflict of interest: None declared

Ethical approval: Not required

\section{REFERENCES}

1. Joglekar SB, Lindvall EM, Martirosian A. Contemporary management of subtrochanteric fractures. Orthop Clin North Am. 2015;46(1):21-35.

2. Tomas J, Teixidor J, Batalla L, Pacha D, Cortina J. Subtrochanteric fractures: treatment with cerclage wire and long intramedullary nail. J Orthop Trauma. 2013;27(7):157-60.

3. Gdoutos EE, Raftopoulos DD, Baril JD. A critical review of the biomechanical stress analysis of the human femur. Biomaterials. 1982;3(1):2-8.

4. Park J, Yang KH. Correction of malalignment in proximal femoral nailing--Reduction technique of displaced proximal fragment. Injury. 2010;41(6):634-8.

5. Kulkarni SS, Moran CG. Results of dynamic condylar screw for subtrochanteric fractures. Injury. 2003;34(2):117-22.

6. Parker MJ, Dutta BK, Sivaji C, Pryor GA. Subtrochanteric fractures of the femur. Injury. 1997;28(2):91-5.

7. Wiss DA, Brien WW. Subtrochanteric fractures of the femur. Results of treatment by interlocking nailing. Clin Orthop Relat Res. 1992;(283):231-6.

8. Suckel AA, Dietz K, Wuelker N, Helwig P. Evaluation of complications of three different types 
of proximal extra-articular femur fractures: differences in complications, age, sex and surviving rates. Int Orthop. 2007;31(5):689-95.

9. Brighton B, Bhandari M, Tornetta P, Felson DT. Hierarchy of evidence: from case reports to randomized controlled trials. Clin Orthop Relat Res. 2003;413:19-24.

10. Park J, Kim SG, Yoon HK, Yang KH. The treatment of nonisthmal femoral shaft nonunions with im nail exchange versus augmentation plating. J Orthop Trauma. 2010;24(2):89-94.

11. Kang SH, Han SK, Kim YS, Kim MJ. Treatment of subtrochanteric nonunion of the femur: whether to leave or to exchange the previous hardware. Acta Orthop Traumatol Turc. 2013;47(2):91-5.
12. Claes L. Mechanobiologie der frakturheilung teil 2. Semantic scholar. 2017;120:23-31.

13. Calori GM, Giannoudis PV. Enhancement of fracture healing with the diamond concept: the role of the biological chamber. Injury. 2011;42(11):1191-3.

14. Tzioupis C, Panteliadis P, Gamie Z, Tsiridis E. Revision of a nonunited subtrochanteric femoral fracture around a failed intramedullary nail with the use of RIA products, BMP-7 and hydroxyapatite: a case report. J Med Case Rep. 2011;5:87.

Cite this article as: Kondewar P, Mahajan N, Gadod L, Sadar A, Atal S. A case of subtrochanteric femur fracture nonunion with failed implant in situ treated with exchange nailing using interlock nail and autologous bone grafting: a case report. Int J Res Orthop 2021;7:864-7. 